\title{
Diferenciación sociodemográfica del espacio urbano de la Ciudad de México
}

Adrián Guillermo Aguilar Instituto de Geografía, Universidad Nacional Autónoma de México

Pablo Mateos Department of Geography, University College London

RESUMEN | El objetivo de este análisis es establecer una diferenciación sociodemográfica del espacio urbano de la Ciudad de México a partir de un metodología que presenta dos principales aportes: el primero es que se aplica una clasificación geodemográfica que muy pocas veces se usa, basada en estilos de vida o niveles socioeconómicos que tienen su origen en la geodemografía o geomárketing; y en segundo lugar, se usa información censal altamente desagregada a nivel de manzana que en muy pocos estudios se han llevado a cabo; como parte de los resultados se identifican seis clusters y se calculan varios índices de segregación residencial. Lo anterior se elabora tomando como referencia los estudios de segregación residencial que se han llevado a cabo en América Latina y en México. Las conclusiones dan cuenta de cómo en la Ciudad de México se notan rasgos del modelo tradicional de segregación, como la persistencia de las élites en ciertos espacios, y cómo los estratos proletarios y las clases medias han tenido una marcada dispersión, junto a la localización periférica de los grupos pobres, más en sintonía con las propuestas del actual modelo de dispersión y fragmentación del espacio urbano.

PALABRAS ClAVE | Metropolización, desigualdad social, fragmentación urbana, segregación, transformaciones socio-territoriales.

ABSTRACT | The aim of this analysis is to establish a socio-demographic differentiation for Mexico City using a methodology that presents two main contributions: first, it applies a geo-demographic typology based on life styles or socioeconomic levels taken from geo-marketing databases; and second, it uses highly disaggregated census information at block levels that has only been applied in very few studies. Six clusters and several segregation indices were calculated. Conclusions show that Mexico City has features of the traditional residential segregation model with elite groups highly concentrated in specific zone, while other socio-demographic groups such as the middle and working classes present a marked dispersion in urban space, more in accordance with the proposals of the new dispersed and fragmented urban model.

KEY WORDS | Metropolization, social inequality, urban fragmentation, segregation, socio-territorial transformations.

Recibido el 13 de noviembre de 2009 , aprobado el 10 de agosto de 2010.

Los autores agradecen la colaboración de Josefina Hernández Lozano en el manejo de las bases de datos y la elaboración de cuadros y mapas, y Alex Singleton (UCL) quien compartió programas para el análisis de clusters y asesoró con respecto a la técnica de clustering $\mathrm{k}$-means.

Correspondencia: Adrián Guillermo Aguilar. E-mail: adrian@servidor.unam.mx | Pablo Mateos.E-mail p.mateos@ucl.ac.uk 


\section{Presentación}

La diferenciación socioeconómica del espacio urbano ha sido un tema de gran interés en el análisis de las ciudades en diferentes contextos culturales, podría decirse que desde el surgimiento de los centros urbanos. Prácticamente todas las ciudades presentan una clara división entre las áreas residenciales ocupadas por los grupos sociales más ricos, aquellas zonas donde predominan las clases medias y los espacios donde los grupos más pobres están confinados. Este proceso de diferenciación es parte esencial del desarrollo urbano y se ha convertido en una línea de investigación en el análisis urbano, entre otras razones, porque la propia existencia de divisiones socioeconómicas en el espacio puede actuar como un mecanismo de reproducción de esas mismas diferencias.

El objetivo de este análisis es establecer una diferenciación socioeconómica del espacio urbano de la Ciudad de México partiendo de una metodología que presenta dos principales aportes. El primero es la aplicación de una clasificación geodemográfica poco común en la literatura en español, basada en estilos de vida o niveles socioeconómicos que tienen su origen en la geodemografía o geomárketing. En segundo lugar, se analiza información censal con una alta desagregación espacial a nivel de manzana, utilizada hasta ahora por muy pocos estudios en México que se centran principalmente en áreas geoestadística básicas (AGEB) con mucho mayor extensión que la manzana. Identificando estas innovaciones, se elabora un análisis de desigualdades socioespaciales, tomando como referencia los estudios de segregación residencial que se han llevado a cabo en América Latina (AL) y en México. Las conclusiones dan cuenta de cómo en la Ciudad de México se perciben rasgos del modelo tradicional de segregación, como, por ejemplo, la persistencia de las élites en ciertos espacios; a la vez, se constatan otros patrones y procesos, como son la marcada dispersión de los estratos proletarios y las clases medias, junto a la localización periférica de los grupos pobres, los cuales están más en sintonía con las propuestas del actualmente prevalente modelo de dispersión y fragmentación del espacio urbano.

\section{Segregación residencial en América Latina. Tendencias recientes}

Los estudios de Segregación Residencial (SR) no muestran ser una línea muy productiva de investigación en la literatura urbana de América Latina. En las últimas dos décadas se observa una ausencia relativa de este tipo de estudios que, partiendo de un análisis sistemático, aporten una visión macro acerca de las principales divisiones sociales en el espacio urbano de las ciudades, naturalmente, salvo algunas notables excepciones. Por un lado, los análisis de SR son escasos, fragmentados y con poca comparación entre los países, por lo que el debate sobre su evolución se basa en experiencias concretas y con frecuencia en el uso de diferentes medidas estadísticas no comparables entre sí. Además ha habido una falta de estudios empíricos sobre este tema que intenten demostrar una hipótesis específica, o hacer una comparación en el tiempo (Rodríguez y Arriagada, 2004, p. 2; Sabatini, 2003, p. 3 ). 
Por otra parte, más allá de un análisis amplio y general de la ciudad se ha otorgado, un mayor énfasis a las condiciones particulares de determinados grupos sociales, en su mayoría los grupos pobres y sus condiciones de asentamiento, y mucho menor a las clases medias o grupos acomodados (Schteingart, 2001, p. 14). Como excepción a esta tendencia general puede mencionarse el caso particular de Santiago de Chile, que cuenta con un número relativamente alto de estudios para todos los grupos sociales $^{1}$ (ver, por ejemplo, Ortiz y Schiappacasse, 2000; Sabatini, Cáceres y Cerda, 2001; Sabatini, 2003; Rodríguez y Arriagada, 2004; Cáceres y Sabatini, 2004; Cortés, 2008; Sabatini y Brain, 2008).

La SR surge como un mecanismo particularmente importante en la reproducción de las desigualdades socioeconómicas, el aislamiento y la inseguridad de los grupos más pobres. Asimismo, la concentración territorial de la pobreza en gran medida influye en la falta de acumulación de capital educativo y social en términos de vínculos sociales, contactos o participación en redes (Rodríguez y Arriagada, 2004, p. 12). En los últimos años han surgido una serie de estudios centrados en temas como los barrios cerrados, la segregación espacial en las áreas metropolitanas, las estructuras policéntricas, la segmentación del mercado de trabajo, el aislamiento de la población urbana pobre o las drogas y la delincuencia. Estos análisis muestran una mayor preocupación acerca de la globalización y la reestructuración económica y sus efectos en las divisiones socioespaciales urbanas, y exigen un trabajo comparativo y empírico a nivel macro - de ciudad- y micro - de barrio.

El concepto de segregación residencial se ha definido como la aglomeración o la distribución de los grupos sociales de una misma condición socioeconómica en el espacio. Esta segregación puede ser identificada de acuerdo a una condición de etnicidad, migración o condición socioeconómica entre las características socioidentitarias más importantes. Se argumenta que en América Latina las fuertes desigualdades de ingresos o el nivel socioeconómico se erigen como el rasgo más característico de la estructura social, incluso más que la pobreza. Con este fin se han diferenciado tres medidas principales de la segregación: (i) la proximidad física entre los espacios residenciales ocupados por distintos grupos sociales, (ii) la homogeneidad social interna de las diferentes divisiones territoriales en que se puede estructurar el espacio urbano, y (iii) la concentración de grupos sociales en zonas específicas de la ciudad (ver Sabatini, 2003, pp. 7-8; Rodríguez and Arriagada, 2004). Sin embargo, existen ciertas dificultades con algunos de estos conceptos. Por ejemplo, una zona totalmente homogénea es prácticamente imposible de encontrar, y depende de la escala del análisis utilizada; los grupos sociales pueden ser altamente segregados en una zona homogénea, lo que supone que los grupos están muy concentrados en lugar de mezclarse. Tampoco está muy claro si la mejor manera de captar la importancia de la distancia entre grupos sociales es a través de su dimensión física, ya que la distancia sociocultural puede ser más importante, si bien es muy compleja de definir y medir (Rodríguez, 2001, p. 13).

En este caso sobresale el trabajo de Francisco Sabatini con un análisis sistemático sobre este tema. 
En el caso de la SR el objetivo principal es diferenciar los estratos socioeconómicos, si bien la manera de identificar las fronteras entre dichos estratos no es tan evidente, como, por ejemplo, en el caso de grupos étnicos. Cabe resaltar que desde la última década en los grandes países de América Latina los datos del censo están disponibles a un nivel altamente desagregado en el espacio urbano. Sin duda, este hecho ha facilitado el análisis a una escala micro en la ciudad, lo que recientemente ha estimulado y aumentado el análisis macro-micro urbano ${ }^{2}$.

\section{La transformación del patrón espacial de la SR}

El proceso más importante en la literatura de las últimas décadas es el cambio en el patrón de la SR observado en las ciudades latinoamericanas. Dicho cambio denota una transformación desde una ciudad relativamente compacta, asociada con el modelo tradicional de SR que dominó hasta la década de 1980, hacia una ciudad con un patrón más disperso ${ }_{2}$ donde las élites se han trasladado a la periferia, han surgido nuevos subcentros urbanos, y los nuevos grupos más pobres se establecieron en una periferia lejana dentro del área rural, entre los procesos más importantes observados (Sabatini, Cáceres y Cerda, 2001; Janoschka, 2002; Borsdorf, 2003; Sabatini, 2003; Sabatini y Cáceres, 2004).

En términos más específicos, el modelo tradicional de la SR se asoció a los siguientes patrones: los grupos de las élites y de alta clase media están situados en un extremo de la ciudad, con una prolongación de su sector que llegaba a la ciudad histórica; grandes zonas de barrios pobres distribuidos principalmente en la periferia, con servicios deficientes; y la presencia de grupos pobres en sectores deteriorados del centro de la ciudad. Una característica interesante del modelo tradicional es que en las zonas residenciales de altos ingresos existe una relativa diversidad social compuesta por clases medias, pero presenciaron ausencia de grupos marginales o informales. Esta estructura urbana se refleja en el modelo propuesto por Griffin y Ford (1980) para las ciudades de AL a finales de la década de 1970 (ver Figura 1), el cual sigue el principio básico de zonas concéntricas mostrando una ciudad con sectores relativamente grandes y homogéneos ${ }^{3}$.

Como consecuencia de dicho modelo de ciudad, las oportunidades de empleo se concentraban en el centro de la ciudad, y la proximidad de residencia con respecto al centro era un símbolo de estatus social. En gran medida el modelo de Griffin y Ford (1980, pp. 406-411) muestra algunas de las siguientes características principales: una economía dinámica en el distrito central de negocios (CBD), una

2 En el caso de la Ciudad de México, por ejemplo, antes del censo de 1990 los datos sólo estaban disponibles a nivel de delegación y/o municipio. Fue en ese año que se incorporó una subdivisión más pequeña a la zona urbana denominada Área Geoestadística Básica (AGEB), que multiplica las unidades de datos disponibles para el espacio de la ciudad. En 2005 la Ciudad de México se dividió en 76 delegaciones y/o municipios, estando integrada por 5.430 AGEB's (véase SEDESOL, CONAPO, INEGI, 2007).

3 Las ciudades latinoamericanas compartían un patrón de calles en damero, desarrollado alrededor de una plaza central, alrededor del cual se agrupan todas las oficinas gubernamentales más importantes, la mayoría de las actividades comerciales y las principales amenidades. 
columna vertebral comercial asociada con un sector residencial de élite, y tres zonas concéntricas de disminución de la calidad residencial: la zona antigua (las mejores residencias), la zona de concentración in situ (residencias de calidad modesta) y la zona de asentamientos precarios periféricos (ver Figura 1).

\section{FIGURA 1 | Un modelo generalizado de la estructura de la ciudad de América Latina}

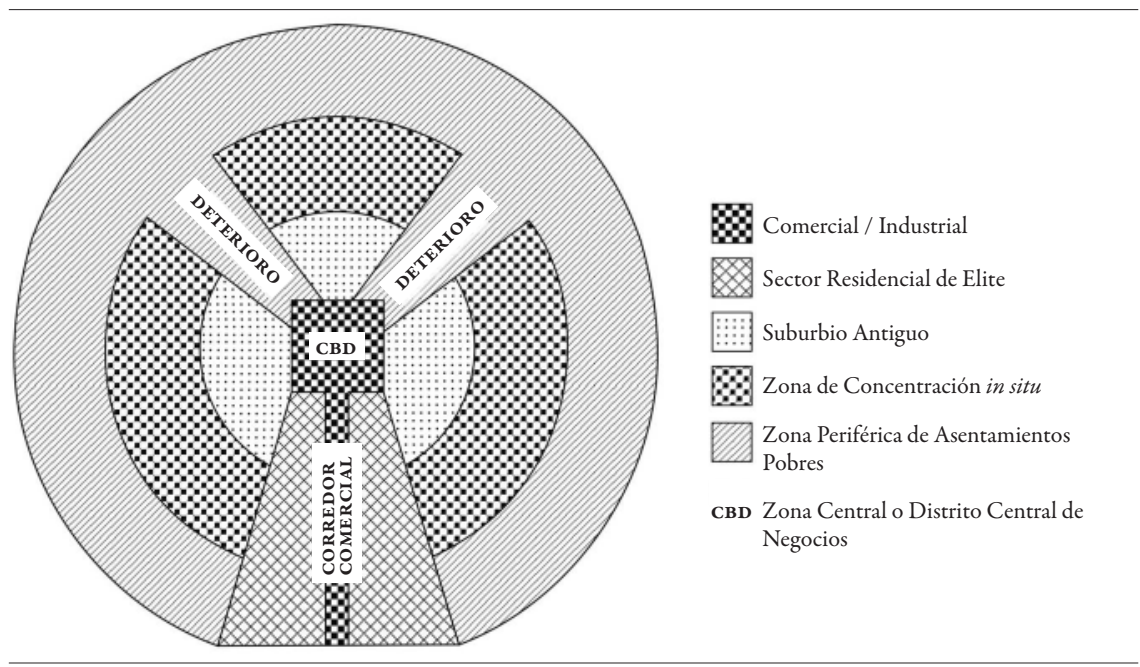

FUENTE GRIFFIN Y FORD (1980).

Los motores de desarrollo urbano y la estructuración del espacio de la ciudad en ese momento (décadas 50-70) eran la industrialización, el liderazgo del Estado en la economía y la planificación urbanística y la migración rural, que contribuyó a un crecimiento urbano acelerado. Las economías nacionales estaban protegidas de la competencia exterior (modelo autárquico), y las ideas políticas tuvieron una fuerte influencia del populismo y el socialismo con una fuerte presencia estatal (Borsdorf, 2003).

La transición al nuevo modelo de dispersión de la SR después de los 80 , muestra las nuevas tendencias en la reestructuración socioespacial del espacio metropolitano. El grado de dispersión de los distintos procesos varía de ciudad en ciudad y de un período a otro y en algunas ciudades la urbanización difusa es más marcada que en otras. El modelo propuesto por Janoschka (2002) es representativo de esta nueva fase (ver Figura 2). En los diferentes estudios realizados en esta etapa destacan tres cambios principales en la ciudades de AL: (i) el desarrollo urbano ha cambiado la escala geográfica de la segregación urbana; (ii) la dispersión de las infraestructuras y las funciones urbanas en el espacio; (iii) la aparición de barrios cerrados como modelo favorecido por los promotores inmobiliarios y clases altas. 
Cada uno de estos cambios se desarrolla brevemente a continuación.

(i) El cambio de escala de la SR. La transformación en curso consiste principalmente en la reducción de la escala geográfica de la segregación en algunas zonas internas de la ciudad y su intensificación en las demás. Este proceso está vinculado a las políticas neoliberales del nuevo modelo económico adoptado durante la década de los 80 . La liberalización del mercado de tierras y la privatización de algunos servicios anteriormente bajo la responsabilidad del Estado dio a los inversionistas privados de bienes raíces un mayor papel en la gestión del espacio urbano. Éstos comenzaron a dispersar sus servicios comerciales y residenciales fuera de los límites tradicionales buscando una manera de capitalizar sus inversiones con un superávit importante a través de formas urbanas más rentables en el mercado urbano. Ejemplos típicos de las formas urbanas desarrolladas bajo este nuevo contexto son; los nuevos centros comerciales ("shopping centers"), barrios cerrados, centros de entretenimiento, altas torres residenciales o urbanizaciones periféricas enormes (ver Figura 2).

Como consecuencia de estos procesos de cambio, los lugares de residencia, trabajo y servicios para los grupos de elite han mostrado una dispersión en el espacio de la ciudad. Los barrios cerrados y los nuevos desarrollos residenciales para clases acomodadas se han localizado incluso dentro del perímetro de los barrios pobres en las zonas más periféricas. Asimismo, los nuevos centros comerciales, y los desarrollos corporativos que representan nuevos subcentros de servicios, han actuado como nuevas "islas de modernidad", estimulando una aparente mayor diversidad social en diferentes áreas de la ciudad (ver Figura 2). Una característica ha sido la concentración de diferentes tipos de capital para la construcción de megaproyectos residenciales, comerciales y de oficinas a diferencia de los pequeños proyectos característicos de la anterior etapa. Los agentes inmobiliarios encontraron una manera de comprar tierras baratas en barrios de clase trabajadora, y ser capaces de vender atractivos desarrollos a las familias de nivel socioeconómico más alto (Sabatini y Cáceres, 2004, pp. 14-19; para el caso de Buenos Aires ver Ciccolella y Baer, 2008, pp. 652 -656).

Espacialmente, el efecto principal es el cambio de escala de la SR; la homogeneidad interna de los nuevos desarrollos residenciales es alta, pero hay una reducción en la distancia física entre los grupos sociales de diferentes estratos socioeconómicos. La SR se hace más intensa en una escala micro a través de bolsas urbanas aisladas entre sí, mientras que a gran escala se exhibe una aparente mayor heterogeneidad y los indicadores de SR se hacen menores. Las nuevas formas urbanas parecen islas de riqueza, de producción, de consumo y de precariedad que se ubican en zonas de carácter más tradicional (Sabatini, 2008, p. 20-21; Janoschka, 2002, pp. 5-26).

La segregación residencial de los grupos más pobres a escala metropolitana o regional es otra consecuencia de la liberalización del mercado de tierras. Aunque algunos grupos pobres se mantuvieron en el espacio interior de la ciudad, otros fueron empujados a trasladarse a la periferia, ya que los valores de la tierra en vez de bajar han aumentado sistemáticamente (Sabatini, Cáceres y Cerda, 2001, pp. 22 y 36). 
FIGURA 2 | El nuevo modelo de ciudad latinoamericana

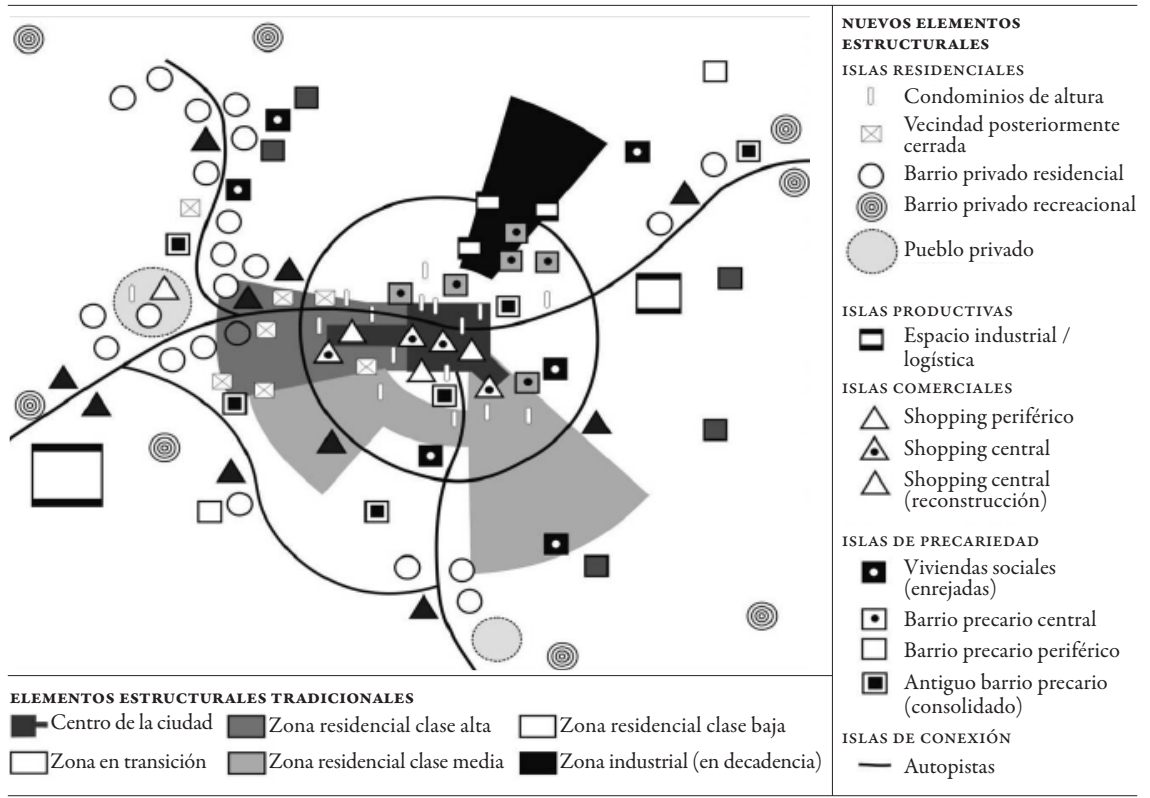

FUENTE JANOSCHKA (2002).

(ii) La dispersión de la infraestructura y las funciones urbanas. Con un patrón más disperso, las áreas suburbanas han sido ocupadas no sólo por los grupos más pobres, sino también por las clases media y rica. Estos nuevos desarrollos periféricos estimularon la construcción de nuevas carreteras, avenidas principales, estaciones de metro y paradas de autobús en las zonas periurbanas. Mientras tanto, los procesos de difusión del consumo y las amenidades fomentaron la presencia de centros comerciales y complejos de entretenimiento creando una nueva división espacial de las funciones urbanas que condujo a un proceso de desconcentración espacial. Los complejos más grandes pueden incluir, además de tiendas exclusivas, actividades de tiempo libre, instituciones educativas privadas y la sede de empresas multinacionales. En este contexto, la formación de una estructura policéntrica en la ciudad se hace más probable cuando "las islas de consumo" se encuentran por doquier.

Una de las consecuencias del nuevo modelo de estructura urbana más disperso es que los centros comerciales no están vinculados únicamente a los barrios ricos, tal y como ocurría en el modelo tradicional (ver Ciccolella y Baer, 2008, pp. 646648) (ver Figura 2). Por este motivo, estos nuevos desarrollos urbanos han sido llamados los "artefactos de la globalización” (ver De Mattos, 1999, y Ciccolella, 1999. Para Santiago de Chile y Buenos Aires respectivamente). Comenzaron bajo los efectos de la reestructuración económica en las grandes ciudades y continuaron con la intensificación del proceso de globalización. Estos constituyen los “símbolos de la 
modernización”, asociados a una actividad productiva más diversificada, nuevos patrones de consumo, aumento de los ingresos del personal ejecutivo y la nueva inversión inmobiliaria en centros corporativos. Los principales ejemplos de ellos son: centros especializados de empresas; nuevos parques industriales o zonas con edificios inteligentes, que representan una mayor verticalización en el espacio urbano; centros comerciales, que ahora tienden a articular los barrios y son la nueva expresión del espacio público, elegantes hoteles y centros de convenciones orientados a las actividades internacionales; núcleos de entretenimiento relacionados con nuevas tecnologías, como cines o parques temáticos; e incluso barrios cerrados.

(iii) Los barrios cerrados. Promotores inmobiliarios en toda AL han adoptado los barrios cerrados como el modelo residencial por excelencia, siendo tan exitoso y omnipresente que ha transformado el espacio metropolitano de diversas maneras. Dicho proceso ha llevado a las familias de las clases alta y media a vivir en barrios populares, lo que en teoría estimula la mezcla social en el espacio a escala micro, y hace que la distancia física entre los grupos más ricos y más pobres se haga más corta (Sabatini y Cáceres, 2004, pp. 16-18). Los barrios cerrados no son necesariamente grandes desarrollos residenciales, aunque en algunas zonas periféricas sí se pueden encontrar, sino generalmente se trata de pequeños desarrollos urbanos que "modernizan" el entorno en una comunidad más grande y en muchos casos de menor condición socioeconómica. Ésta es, por tanto, la expresión en formas urbanas de la reducción de escala en el proceso de la SR a la que nos referíamos anteriormente (ver Figura 2).

Los barrios cerrados crean espacios en los que aun estando los grupos sociales más cercanos entre sí se encuentran separados por paredes, vallas perimetrales y tecnologías de seguridad. Sus habitantes tienden a no circular a pie o interactuar en áreas comunes; estos nuevos espacios de la segregación espacial pueden ser considerados como "enclaves fortificados", porque son espacios que se privatizan, se encierran y se monitorean para el consumo, ocio y trabajo (Caldeira, 2000, p. 213). Algunos autores sostienen que la principal justificación para la proliferación de barrios cerrados es el desplazamiento de las clases altas del centro de la ciudad y el temor a la delincuencia que ha hecho a la gente de todas las clases sociales buscar formas más seguras de residencia (ver Caldeira, 2000, p. 254). Sin embargo, otros autores sostienen que los principales motivos para mudarse a vivir a una comunidad cerrada no están relacionados con la exclusión social, ni el temor a la delincuencia, sino a la relación precio-calidad de la vivienda, por las altas expectativas del incremento del precio de la propiedad y el acceso a bienes que de manera individual son difíciles de conseguir, como son las amenidades ambientales, instalaciones deportivas, educativas y de seguridad (véase Sabatini y Cáceres, 2004, p. 19).

Cabe también mencionar que los barrios cerrados simbolizan un nuevo estilo de vida asociado a propiedades aisladas con mejor infraestructura y servicios. Tal y como Caldeira (2000, p. 258) indica, "los enclaves fortificados confieren estatus, la construcción de símbolos de estatus es un proceso que profundiza las diferencias sociales y crea los medios para la afirmación de la distancia social y la desigualdad". 
Así, los barrios cerrados surgen como un nuevo paradigma de desarrollo urbano y un modelo de segregación social en el espacio de la ciudad, que va en aumento. Este modelo es tolerado por las autoridades locales, favorecido por los promotores inmobiliarios y aceptado por los residentes. Junto a la segregación social hay otros dos elementos fundamentales que emergen con ellos: la fragmentación y la privatización (Solinis, 2002, pp. 21-23). Por un lado, la fragmentación del espacio urbano a escala micro queda favorecida con la creación de estos enclaves residenciales de clase alta en un entorno de zonas de nivel socioeconómico más bajo. Por otro, se propicia la privatizacion de las calles y de otros espacios públicos, lo cual lleva a los residentes de barrios cerrados a separarse del espacio público y con ello a disminuir la interacción social en la ciudad, porque las calles y plazas abiertas están cada vez más marcadas por el temor y el miedo.

Todas las transformaciones urbanas mencionadas hasta ahora han activado claramente el debate sobre la estructura urbana y la distribución de las actividades productivas en las ciudades de América Latina. El modelo de ciudad trazado alrededor de los círculos concéntricos y sectores se ha puesto en duda, rechazado en base al análisis de los fenómenos recientes de discontinuidades urbanas y la atomización de unidades funcionales. El proceso de metropolización y el impacto de la globalización han dado un impulso a las nuevas divisiones urbanas de la ciudad, con la aparición de recientes transformaciones y procesos como la gentrificación, subcentros urbanos, barrios cerrados, centros comerciales, y un aumento de la heterogeneidad dentro de los barrios consolidados. Además, tal y como señalan Schapira y Cattaneo (2008, p. 88), la periferia aparece como un gran espacio fragmentado con un patrón de urbanización de células más o menos autónomas, como resultado de una acción política dominada por la intermitencia y los conflictos, donde los enclaves residenciales surgen de manera dispersa. En otras palabras, el nuevo modelo de dispersión y fragmentación se sobrepone al modelo tradicional de las zonas concéntricas y sectoriales, y desde esta perspectiva el análisis de la ciudad en términos de fragmentación o división urbana a pequeña escala parece más adecuado que la tradicional lógica centro-periferia.

\section{El análisis sociodemográfico de la Zona Metropolitana de la Ciudad de México}

A principios de la década de los 70, el análisis social de áreas (Shevky y Bell, 1955), que emanaba del análisis factorial urbano anteriormente descrito, cayó en desuso como herramienta de investigación social en urbanismo, al considerarse demasiado descriptiva y desvinculada de las corrientes de pensamiento que prevalecían en aquella época en las ciencias sociales. No obstante, desde principios de los 80 el interés por la clasificación de ciudades en zonas de "estilos de vida" quedó íntimamente ligado a la función de marketing de las empresas privadas líderes, particularmente las de distribución comercial. Con el tiempo, este tipo de análisis se erigió como una metodología propia denominada geodemografía o geomárketing (Harris et al., 2005), que con los años ha vuelto a ser utilizada en círculos académicos, así como en numerosas aplicaciones en el sector público (Longley y Mateos, 2006). 
La metodología de la clasificación geodemográfica se basa en clasificar zonas residenciales muy pequeñas de la ciudad (generalmente manzanas o segmentos de calles) en una tipología multidimensional de estilos de vida. Para ello se utilizan datos provenientes del censo de población, encuestas y registros administrativos. Con estos y mediante técnicas de clustering, se clasifican dichas zonas en grupos o clusters según su cercanía en el espacio de variables seleccionado. Una vez que estos clusters son evaluados, se procede a su descripción en cuanto a los estilos de vida característicos de sus habitantes y a su ulterior análisis espacial o en conjunción con otras variables (para una explicación completa del funcionamiento de dichas técnicas ver Harris, 2005).

En los estudios sobre segregación residencial en América Latina descritos en la sección anterior, las tipologías socioeconómicas en la base de dichos estudios se elaboran mediante análisis factorial. Este consiste básicamente en la reducción de las muchas variables tenidas en cuenta a tres o cuatro dimensiones principales, generalmente ingresos, nivel educativo, ocupación y vivienda. Después, calculan diversos índices de segregación residencial para cada una de dichas dimensiones o factores de manera independiente, como si los aspectos que las entrelazan entre sí y en el espacio no tuvieran especial relevancia. Frente a esta simplificación, la realidad socioeconómica de las ciudades latinoamericanas se erige con una complejidad mucho mayor, en la que a estas variables socioeconómicas básicas se aúnan aspectos como la estructura de edad de la población, composición de hogar, antigüedad y tipología constructiva del barrio o colonia, migración, balance de género, densidad de población, o incluso distancia al centro de la ciudad, aspectos todos ellos que marcan ciertos "estilos de vida" residenciales. Además, todos los estudios abordan la medición de la segregación residencial tomando como base zonas administrativas relativamente extensas, como distritos o zonas básicas estadísticas como las mencionadas AGEB. Estas unidades no reflejan necesariamente las zonas reales de preferencia residencial que operan en el mercado de la vivienda en Latinoamérica, como por ejemplo las colonias y desarrollos residenciales concretos, y que determinan los patrones tan contrastados que encontramos en nuestras ciudades.

\section{El análisis geodemográfico}

A partir del planteamiento anterior, proponemos aquí la utilización de una clasificación geodemográfica para el análisis de la segregación residencial para la Zona Metropolitana de la Ciudad de México (ZMCM), partiendo de información censal altamente desagregada en el espacio: el Censo de Población y Vivienda de 2000 a nivel de manzana para todo el espacio metropolitano. Mediante la clasificación de unidades espaciales pequeñas de la Ciudad de México se puede capturar con mayor precisión la complejidad de los estilos de vida que sus pobladores tienen en común (un total de 17.957 .062 residentes en el Censo de 2000) ${ }^{4}$. Esto es ahora posible al tener acceso a datos censales a nivel de manzana que con 142.291 unidades y un 
promedio de 126,1 residentes son mucho más homogéneas en cuanto a sus características geodemográficas y estilos de vida que los AGEB (4.974 unidades y promedio de 3.610,2 residentes). Para este análisis se elaboró un cuadro con conteos absolutos para 170 variables del Censo de Población y Vivienda 2000 en la ZMCM desagregados a nivel de manzana (142.291 unidades) 5 .

En cuanto al procedimiento metodológico, en primer lugar se procedió a la elaboración de porcentajes y ratios a partir de los conteos absolutos, así como a la estandarización de los datos. Para la mayoría de las variables se calcularon porcentajes sobre la población total en la manzana, mientras que algunas variables exigían un denominador distinto, como, por ejemplo, el número de hogares, de viviendas o de personas de cierto género o grupo de edad. La estandarización de los datos en una escala común es necesaria para que la clasificación de manzanas sea efectiva, y las "distancias" entre variables sean medidas en las mismas unidades. Para ello se estandarizaron las variables calculado $z$-scores o, lo que es lo mismo, computando la diferencia entre un valor dado y la media de la variable partida por la desviación estándar de la variable. Por definición, los z-scores resultantes tienen todos una media de 0 y desviación típica de 1 y por tanto una distribución de frecuencias que puede ser comparada en igualdad de condiciones (Robinson, 1998).

En segundo lugar, se seleccionaron las variables más adecuadas para llevar a cabo el clustering de manzanas. En este tipo de clasificaciones es preferible seleccionar unas pocas variables que aporten información clave en la discriminación entre tipologías de barrios, sin solaparse, antes que inundar la clasificación con variables altamente correlacionadas entre sí. Con este fin se procedió a elaborar una matriz de correlaciones entre las 170 variables estandarizadas, para evaluar en qué medida la variación de éstas a través de las 142.291 manzanas apunta en la misma dirección. Dicha matriz de correlación se utilizó posteriormente para informar el proceso de selección de variables. Para ello se llevó a cabo un análisis de componentes principales (PCA) en dicha matriz de correlaciones, dando como resultado tres primeros componentes que explican el $84,5 \%$ de la varianza. También se elaboró un árbol expandido mínimo (o minimum spanning tree, en adelante MST), obteniendo la relación entre cada variable y sus variables más cercanas en la matriz de correlaciones (relación tanto positiva como negativa), siguiendo la técnica propuesta en Harris (2005, p. 155). Sorprendentemente, la variable que está más relacionada con todas las demás, y que por lo tanto aparece en el centro del MST, es el número de ocupantes por cuarto. De esta variable emanan una serie de "ramas", de las que cabe destacar ocho que apuntan en direcciones distintas, ramificándose a su vez en otras direcciones. Del análisis de componentes principales se extrajeron las variables más cercanamente relacionadas con los tres primeros componentes, mientras que del MST se extrajeron las variables clave en cada rama principal y las "bifurcaciones" más importantes, seleccionando variables con baja correlación entre sí. Esto resultó en un total de 34 variables únicas que aparecen en el Cuadro 1 y que constituyeron la base para posteriores análisis.

5 Agradecemos al Instituto Nacional de Estadística y Geografía (INEGI) por habernos facilitado la base de datos correspondiente para elaborar este análisis. 
CUADRO 1 | Lista de las 36 variables seleccionadas para el clustering

\begin{tabular}{|c|c|c|}
\hline VARIABLE INEGI & NOMBRE CORTO & DESCRIPCIÓN \\
\hline & Densidad & Densidad de Población \\
\hline $\mathrm{Z} 1$ & Tot_Pob & Población total \\
\hline $\mathrm{Z7}$ & Pob_0_14 & Población de 0 a 14 años \\
\hline Z42 & Pob_60_mas & Población de 60 años y más \\
\hline Z48 & Rel_depend & Relación de dependencia \\
\hline Z49 & Rel_hijos_mujer & Relación niños-mujer \\
\hline $\mathrm{Z} 51$ & Pob_sin_seg_salud & Población sin derechohabiencia a servicio de salud \\
\hline $\mathrm{Z} 52$ & Pob_c_IMMS & Población derechohabiente al IMSS \\
\hline $\mathrm{Z} 55$ & Pob_nac_fuera & Población nacida fuera de la entidad \\
\hline $\mathrm{Z} 78$ & Pob_inst_medysup & Población de 15 años y más con instrucción media superior o superior \\
\hline Z82 & Pob_inst_sup & Población de 18 años y más con instrucción superior \\
\hline Z83 & Años_escol & Grado promedio de escolaridad \\
\hline Z102 & Pob_inactiv & Población económicamente inactiva \\
\hline Z106 & Ocup_secund & Población ocupada en el sector secundario \\
\hline Z107 & Ocup_terciar & Población ocupada en el sector terciario \\
\hline Z109 & Ocup_jornal & Población ocupada como jornalero o peón \\
\hline $\mathrm{Z} 110$ & Trab_cuent_prop & Población trabajadora por cuenta propia \\
\hline Z114 & Ocup sin ingr & Población ocupada que no recibe ingreso por trabajo \\
\hline Z115 & Ocup_<1_salario & $\begin{array}{l}\text { Población ocupada que recibe menos de un salario mínimo mensual de } \\
\text { ingreso por trabajo }\end{array}$ \\
\hline $\mathrm{Z} 118$ & Ocup_>5_salarios & $\begin{array}{l}\text { Población ocupada que recibe más de } 5 \text { salarios mínimos mensuales de } \\
\text { ingreso por trabajo }\end{array}$ \\
\hline $\mathrm{Z} 121$ & Viv_mat_ligeros & Viviendas particulares con techos de materiales ligeros, naturales y precarios \\
\hline $\mathrm{Z} 128$ & Viv_un_cuarto & Viviendas particulares con un solo cuarto (cuarto redondo) \\
\hline Z129 & Viv_2_5_cuartos & Viviendas particulares con 2 a 5 cuartos (incluye cocina exclusiva) \\
\hline Z130 & Viv_1_dorm & Viviendas particulares con un dormitorio \\
\hline Z133 & Viv_coc_no_excl & Viviendas particulares con cocina no exclusiva \\
\hline Z137 & Viv_dren_no_red & $\begin{array}{l}\text { Viviendas particulares con drenaje conectado a fosa séptica, barranca o } \\
\text { grieta, río, lago y mar }\end{array}$ \\
\hline Z138 & Viv_sin_dren & Viviendas particulares sin drenaje \\
\hline $\mathrm{Z} 148$ & Viv_prop & Viviendas particulares propias \\
\hline Z149 & Viv_prop_pagad & Viviendas particulares propias pagadas \\
\hline Z158 & Viv_c_boiler & Viviendas particulares que disponen de calentador de agua (boiler) \\
\hline $\mathrm{Z} 160$ & Viv_c_compu & Viviendas particulares que disponen de computadora \\
\hline $\mathrm{Z} 161$ & Viv_c_todos_bien & Viviendas particulares con todos los bienes \\
\hline $\mathrm{Z} 163$ & Ocup_p_viv & Promedio de ocupantes en viviendas particulares \\
\hline Z164 & Ocup_p_cuart & Promedio de ocupantes por cuarto en viviendas particulares \\
\hline $\mathrm{Z} 167$ & Hog_jef_fem & Hogares con jefatura femenina \\
\hline $\mathrm{Z} 169$ & Pob_hog_jef_mas & Población en hogares con jefatura masculina \\
\hline
\end{tabular}

FUENTE ELABORACIÓN PROPIA. 
En tercer lugar, se llevó a cabo la clasificación de manzanas mediante una técnica de clustering denominada k-means. Esta es una técnica inductiva de clasificación y es la más utilizada para elaborar clasificaciones geodemográficas (Harris et al., 2005). Se trata de una metodología que parte de una división imperfecta de las unidades a clasificar (en este caso manzanas) en un número de clusters predeterminado, y a partir de la misma se proponen movimientos de unidades entre clusters a través de múltiples iteraciones hasta encontrar el resultado óptimo que minimiza las diferencias internas en cada cluster (en cuanto a sus "distancias" en el espacio k-dimensional de variables) y maximiza las diferencias entre clusters. Por ejemplo, esta fue la técnica utilizada para la elaboración, entre otras, de la clasificación geodemográfica oficial del Reino Unido, denominada OAC (Vickers y Rees, 2007). Para el análisis aquí descrito se tomó un número de seis clusters para clasificar las 142.291 manzanas, adoptando un número de estratos socioeconómicos habitualmente propuesto en la literatura mencionada, por ejemplo por Rubalcava y Schteingart (2000b). Se utilizó un algoritmo de k-means ponderado, mediante el cual se pondera cada variable para cada manzana según la proporción de población de la manzana respecto a toda la ciudad. De esta manera se asegura una combinación equitativa de las variables analizadas independientemente del tamaño de la población de cada unidad de análisis (la manzana). La técnica de k-means se parametrizó con 100 iteraciones para llegar a una solución óptima de división en seis clusters. No obstante, para asegurarnos que obtenemos la mejor solución posible, minimizando la posibilidad de caer en un "mínimo local", se programó una rutina que repitió el proceso de clustering de manera independiente 1.000 veces. Es decir, se repitieron 1.000 análisis independientes de clustering, cada uno con 100 iteraciones del algoritmo $k$-means. Finalmente se tomó la solución óptima de entre las 1.000 alcanzadas, definida por aquella que mejor minimizó la raíz cuadrada de la suma del cuadrado de las diferencias dentro de cada cluster. El resultado final fueron seis clusters con un tamaño de población total en el mismo orden de magnitud, y similar número de manzanas asignadas a cada uno, excepto el cluster 5 que presenta un bajo número de manzanas muy pobladas (ver Cuadro 2).

CUADRO 2 | Tamaños de los seis clusters seleccionados

(población y número de manzanas)

\begin{tabular}{cccc}
\hline CLUSTER & NO. MANZANAS & POBLACIÓN TOTAL & POBLACIÓN MEDIA POR MANZANA \\
\hline 1 & 17.952 & 2.055 .797 & 114,5 \\
\hline 2 & 19.743 & 1.587 .619 & 80,4 \\
\hline 3 & 49.504 & 6.816 .571 & 137,7 \\
\hline 4 & 18.618 & 2.234 .257 & 120 \\
\hline 5 & 993 & 1.067 .682 & 1075,2 \\
\hline 6 & 34.927 & 4.152 .252 & 118,9 \\
\hline Total & $\mathbf{1 4 1 . 7 3 7}$ & $\mathbf{1 7 . 9 1 4 . 1 7 8}$ & $\mathbf{1 2 6 , 4}$ \\
\hline
\end{tabular}

FUENTE ELABORACIÓN PROPIA. 


\section{Interpretación de la clasificación geodemográfica de la ZMCM}

En las Figuras 3 y 4 están representados los valores medios ponderados de las 34 variables seleccionadas para el clustering, para cada uno de los seis clusters resultantes ( $z$-score medio por cluster ponderado por la población de la manzana). Estos gráficos de radar permiten establecer una comparación visual rápida entre los seis clusters, así como con la media de toda la ciudad, que en este caso tiene un valor cero (como resultado de la estandarización por medio de $z$-scores antes mencionada) y aparece en el centro con línea continua delgada. Los posibles valores de las variables estandarizadas fluctúan entre un máximo de 2 y un mínimo teórico de -2 . Estos valores representan la media para cada cluster del número de desviaciones estándar de las manzanas con respecto a la media de la variable para toda la ZMCM. Es decir, por ejemplo, en la Figura 3, el Cluster 4 tiene una media de 2 desviaciones estándar por encima de la media para la ZMCM de la variable "Población con Instrucción Superior", mientras que el valor más bajo para esta variable corresponde al Cluster 1 (Figura 4) que presenta 0.7 desviaciones estándar por debajo de dicha media. Asimismo, en las Figuras 5, 6 y 7 se ofrece un resumen de la distribución geográfica de estos seis clusters a nivel de manzana para toda la ZMCM (un mapa por cluster).

FIGURA 3 | Gráfico de radar con variables seleccionadas: Clusters 2, 3 y 4

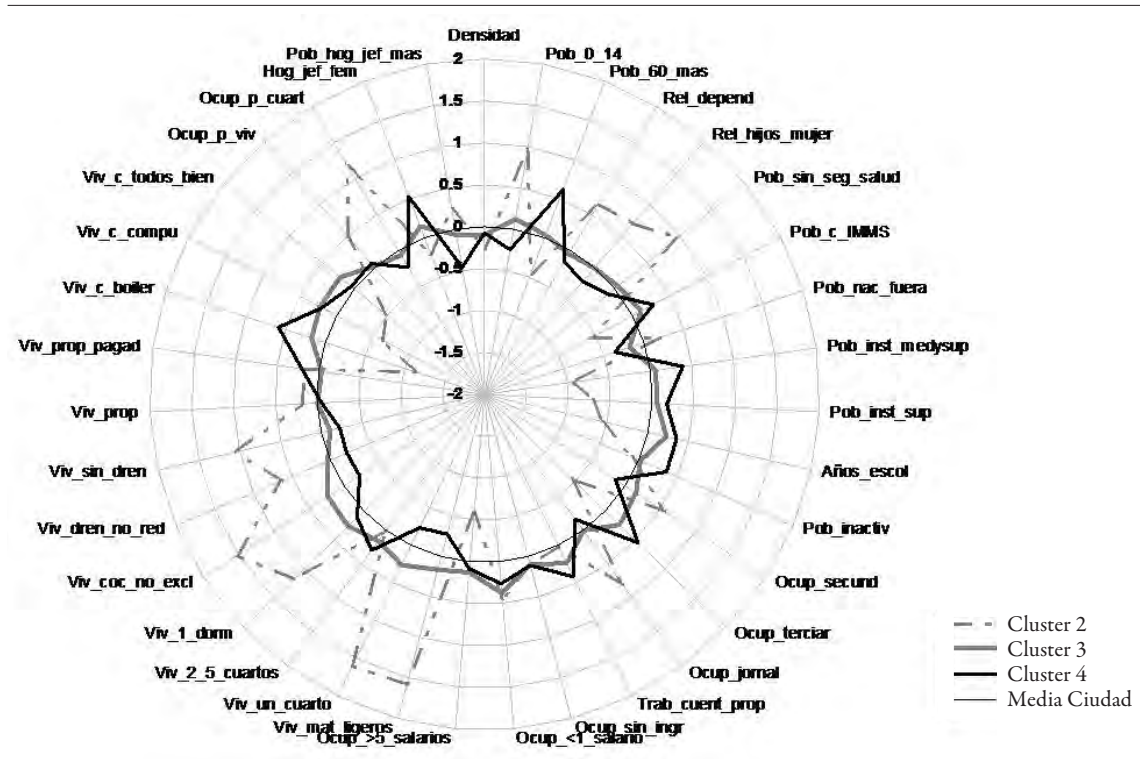

FUENTE ELABORACIÓN PROPIA. 
FIGURA 4 | Gráfico de radar con variables seleccionadas: Clusters 1, 5 y 6

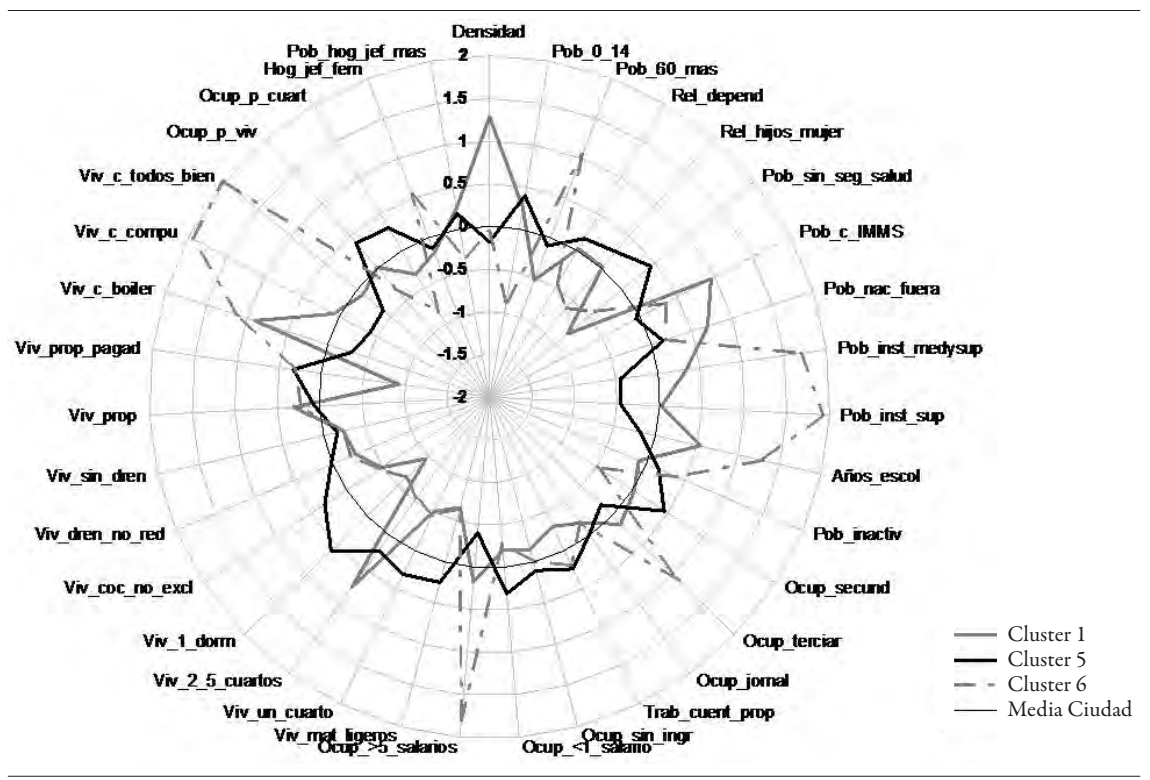

FUENTE ELABORACIÓN PROPIA.

Mediante estos gráficos de radar y mapas se ha procedido a hacer una somera interpretación de las características sociodemográficas de la población que reside en cada uno de estos seis clusters. En los próximos párrafos se ofrece una breve descripción de cada cluster, así como una primera propuesta de nombre corto que resuma su principal característica:

\section{Cluster 1: Periferia urbano-rural marginal}

Presenta una estructura de población muy joven, con una alta relación de hijos por mujer, alto peso de migración interna, bajo porcentaje de instrucción media y superior, alto porcentaje de población ocupada en el sector secundario y como jornaleros, y un alto porcentaje de población sin seguro de salud y muy baja pertenencia al IMSS. Este cluster presenta una distribución de salarios muy sesgada hacia la banda de menos de un salario mínimo. La población de este cluster reside principalmente en viviendas en propiedad pero construidas con materiales ligeros y predominantemente con un solo dormitorio, lo que deriva en una altísima tasa de ocupación por cuarto, en viviendas sin cocina exclusiva y sin acceso a red de drenaje, así como carentes de los bienes indicativos básicos. La distribución espacial de este cluster está caracterizada por una extrema periferización en los márgenes de la ZMCD, en zonas en contacto con la periferia rural, principalmente del Estado de México. Colonias representativas de este cluster son: Plan de Ayala (Naucalpan), Zapotitla y San Andrés Mixquic (Tláhuac), Industrial Lechería (Tultitlán), Chicoloapan y San Miguel Topilejo (Tlalpan). 


\section{Cluster 2: Empleados de oficina en unidades habitacionales}

Los habitantes de este cluster viven en manzanas con una densidad de población altísima, lo cual denota un predominio de torres de viviendas o unidades habitacionales. Su estructura de población es relativamente joven, aunque con una relación de hijos por mujer cercana a la media, y cuenta con un alto componente de migrantes internos. La mayoría de la población cuenta con seguro de salud, principalmente proporcionado por el IMSS, y trabaja mayoritariamente en actividades terciarias por cuenta ajena (tiene un bajo índice de trabajo por cuenta propia). La distribución de salarios predominante es entre uno y cinco salarios mínimos, con un cierto sesgo hacia la banda alta de este rango. El nivel de educación es alto en relación a la media, principalmente con instrucción media, y presenta un nivel de años de escolaridad superior a la media. La tipología de viviendas que ocupan es en general de calidad, tipo departamento, principalmente en propiedad pero que aún no están pagados, contando con boiler y computadora entre los bienes indicativos. El número de ocupantes por cuarto es bajo, y los hogares están principalmente encabezados por hombres. Su distribución espacial está muy concentrada en áreas de alta densidad poblacional en el interior de la ZMCM, tanto en el Distrito Federal como en núcleos urbanos de los municipios del Estado de México. Colonias representativas de este cluster son: Nuevo Valle de Aragón (Ecatepec), Lomas de Santa Fe (Cuajimalpa), Fuentes del Valle y Bosques de la Hacienda (Cuautitlán) y El Rosario (Azcapotzalco).

\section{Cluster 3: Proletariado periférico}

Está caracterizado por una estructura de población joven y alta relación de hijos por mujer, con cierta presencia de migrantes internos. Exhibe un porcentaje elevado de personas sin seguro de salud, y una pertenencia al IMSS a la par con la media de la ciudad. El nivel de instrucción predominante es la escuela primaria, y el número de años de escolaridad está por debajo de la media. La estructura ocupacional está caracterizada por empleo en el sector secundario, en gran grado por cuenta propia, y también presenta una alta proporción de población inactiva. La proporción de personas ocupadas con ingresos por debajo de un salario mínimo está por encima de la media, y la que recibe más de cinco salarios es la más baja de los seis clusters. Ocupa una tipología de viviendas en propiedad y por lo general ya pagadas, aunque de baja calidad constructiva, con drenaje pero no conectado a la red pública, y con cierta variabilidad de tamaños, si bien predominan las viviendas de un solo cuarto. El número de ocupantes por cuarto y por vivienda es alto y la dotación de bienes indicativos está claramente por debajo de la media. Su distribución espacial está repartida en los núcleos urbanos de toda la periferia, principalmente en el Estado de México, aunque en manzanas con baja densidad de población. Colonias representativas de este cluster son: San Lorenzo Atemoaya (Xochimilco), Ixtapaluca (Edomex), San Pablo de las Salinas (Tultitán), Torres de Padierna (Tlalpan), La Magdalena Chichicaspa (Huixquilucan) y Ciudad Netzahualcóyotl.

\section{Cluster 4: Élites urbanas}

Este cluster es muy distinto a todos los demás. Presenta una estructura de población muy envejecida, con baja relación de hijos por mujer. Aunque una buena parte de la población no es derechohabiente de un seguro de salud, el grado de pertenencia al IMSS está por encima de la media. El nivel de instrucción superior es muy alto así como el prome- 
dio de años de escolaridad. La proporción de población económicamente inactiva es alta, y la mayoría de las personas ocupadas trabaja en el sector terciario, en cierta medida por cuenta propia, con ingresos predominantes por encima de cinco salarios mínimos. Las viviendas son de buena calidad y gran tamaño, generalmente en propiedad y además ya pagadas, con presencia de todos los bienes indicativos. El ratio de ocupantes por vivienda y por cuarto es muy bajo, y el porcentaje de hogares encabezados por mujeres es alto. Su distribución espacial está claramente concentrada en el Distrito Federal, especialmente en la mitad occidental. Colonias representativas de este cluster son: Lomas de Chapultepec (Miguel Hidalgo), Tlatelolco (Cuauhtémoc), Lomas de Tecamachalco (Naucalpan), Altavista (Álvaro Obregón) y Ciudad Satélite (Naucalpan).

\section{Cluster 5: Zonas mezcladas}

Este cluster representa muy bien la media de toda la ciudad. Más que tratarse de familias típicas, lo que parece que está reflejando es la presencia de manzanas que abarcan un área muy grande, incluyendo a mucha población de todo tipo en su interior. Mientras que la media de personas por manzana en toda la ciudad es de 126 habitantes, cifra alrededor de la cual oscilan los demás clusters, el cluster 5 tiene una media de 1.075 habitantes por manzana, con solo 993 manzanas (ver Cuadro 2). Por todo ello, este cluster está caracterizado por representar la media de la ciudad a través de zonas muy grandes y socioeconómicamente diversas, situadas principalmente en la periferia del Distrito Federal en zonas de laderas o en torno a los intersticios vacíos urbanos en el interior de la ciudad. Colonias representativas de este cluster son: Valle de Tepepan (Tlalpan), San Bartolo Ameyalco (Álvaro Obregón), Santa Cruz Acayucan (Azcapotzalco) y Ampliación Petrolera (Azcapotzalco).

\section{Cluster 6: Clase media educada}

Éste presenta una estructura de edad joven pero, a diferencia de otros clusters, con una baja relación de hijos por mujer, y una baja relación de dependencia, lo cual denota una gran presencia de población en edad activa y una tasa de fertilidad por debajo de la media. Es el cluster con menor presencia de migrantes internos, es decir, con una alta tasa de natalidad en la ZMCM. Presenta una cobertura de seguro de salud cercana a la media, con un alto grado de pertenencia al IMSS. El nivel educativo está claramente por encima de la media de la ciudad, en cuanto a años de escolaridad, así como instrucción media y superior, prácticamente a la par del Cluster 2, aunque por debajo del Cluster 4. La ocupación principal es en el sector terciario, y gran medida por cuenta propia (a diferencia del Cluster 2). La distribución de ingresos es cercana a la media, pero con un ligero sesgo hacia la banda de menos de un salario mínimo. Predominan las viviendas en propiedad ya pagadas, cuya calidad es mejor que la media, y de tamaño grande (de dos a cinco cuartos), por lo que el ratio de ocupantes por cuarto es bajo. Las viviendas suelen contar con computadora y boiler. El porcentaje de hogares encabezados por mujeres es superior a la media. Su distribución espacial es dispersa por toda la ZMCM, pero no periférica, ubicándose en zonas económicamente accesibles cercanas a los principales centros de actividad económica. Colonias representativas de este cluster son: Industrial Vallejo (Azcapotzalco), Escandón (Miguel Hidalgo), Nonoalco (Benito Juárez), Tránsito (Cuauhtémoc), Pedregal del Maurel y Copilco El alto (Coyoacán) y Bosques de la Hacienda (Cuautitlán). 
FIGURAS 5, 6,7|ZMCM, Diferenciación socioeconómica. Proyección UTM zona 14

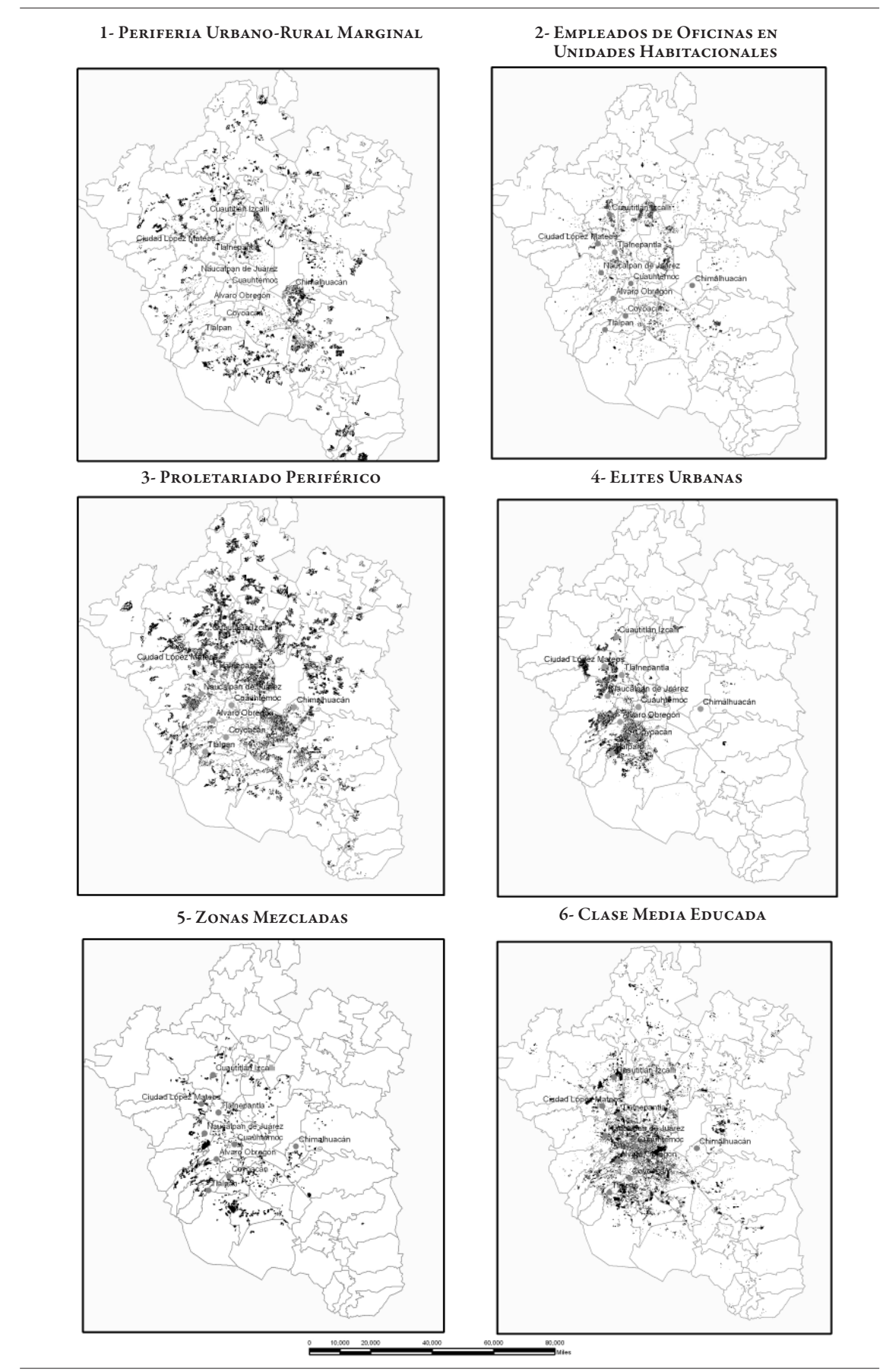

FUENTE ELABORACIÓN PROPIA. 


\section{Análisis de índices de segregación residencial entre clusters geodemográficos}

En esta última sección se describe el cálculo de índices de segregación para los seis clusters geodemográficos descritos.

Como base del análisis de segregación socioeconómica se tomaron los tipos de manzanas, divergiendo de la mayor parte de la literatura de segregación en Latinoamérica que se basa en los individuos u hogares. Es decir, una vez asignados a uno de los seis clusters geodemográficos, todos los individuos y hogares de cada manzana adoptan las mismas características de la manzana en la que residen. Aquí estamos asumiendo que la tipificación hecha de cada manzana representa bien las características de todos o la mayoría de sus residentes, aunque somos conscientes de que esto puede ser evidentemente cuestionado mediante la crítica de la "falacia ecológica". Para medir el grado de segregación de estos clusters geodemográficos dentro de la ciudad, las manzanas como miembros de dichos clusters han de ser comparadas con sus manzanas vecinas para asesorar el grado de segregación espacial entre clusters en un área de contacto local. Para demarcar dicho ámbito de "vecindad local" se han agrupado las manzanas por el AGEB al que pertenecen. Es decir, en el Censo 2000 de la ZMCM tenemos 142.291 manzanas y 4.974 AGEBs, lo cual arroja una media por AGEB de 28,6 manzanas y 3.601 habitantes. Por tanto, a partir de aquí la unidad de análisis espacial es el AGEB, para el que se calculó el número de personas en cada AGEB que pertenece a cada uno de los seis clusters geodemográficos. Si la distribución de dichos clusters fuese equitativa en cada AGEB de la ciudad, no existiría segregación, mientras que, por el contrario, si dicha distribución es desigual esperamos encontrar ciertos niveles de segregación socioeconómica.

Se calcularon una serie de índices de segregación residencial que frecuentemente aparecen en la literatura (Apparicio et al., 2008) ${ }^{6}$, tratando de capturar el mayor número de dimensiones de este fenómeno (Massey y Denton, 1988). El índice comúnmente utilizado para resumir el grado de segregación de un grupo o una ciudad es el índice de Disimilaridad $(D)$ (Duncan y Duncan, 1955), que mide la dimensión de similaridad (evenness). Éste puede interpretarse como la proporción de residentes que tendría que moverse de zona para que el grupo o la ciudad completa esté distribuido de manera uniforme (Massey y Denton, 1988). La disimilaridad $D$ de toda la ZMCM para los seis clusters tomados en su conjunto es de 0.73 , lo que quiere decir que el 73\% de los habitantes tendría que cambiar de AGEB para que la ciudad no presentara segregación alguna. En general, se considera que valores entre 0.3 y 0.6 simbolizan un nivel de segregación media y por encima de 0.6 un nivel muy segregado. Por tanto, en este indicador la ZMCD presenta un patrón muy segregado.

En el Cuadro 3 se presentan los valores de cinco índices distintos para cada uno de los seis clusters. Los cuatro primeros índices miden cuatro de las dimensiones de segregación propuestas por Massey y Denton (1988), de izquierda a derecha: similaridad, aislamiento, concentración y clustering (no se calculó cen-

El cálculo se llevó a cabo mediante el software Segregation Analyser (Apparicio et al 2008). 
tralidad) mientras que el último (en la columna más a la derecha) mide el nivel de entropía en una dimensión adicional usualmente denominada diversidad (Simpson, 1949). Para más detalles acerca de las ecuaciones e implantación adoptada de dichos índices ver Apparicio et al. (2008). Es necesario recalcar que los resultados de dichos índices hacen referencia al nivel de segregación entre tipos de manzanas - o clusters geodemográficos- y no de individuos u hogares. Esta aclaración ha de tenerse en cuenta al comparar los valores de dichos índices presentados con la literatura previa en esta área citada en la primera parte del artículo. Como se puede apreciar en el Cuadro 3, prácticamente todos los clusters presentan un nivel de segregación medio-alto o alto en casi todas los índices (los valores máximos aparecen en negrita). El Cluster 4 que hemos denominado Élites Urbanas, es el más segregado del resto de la población en tres de estos cinco índices $(\mathrm{D}=0.86, \mathrm{Px}=0.71$, $\mathrm{H}=0.68)$. Se trata del cluster con mayor nivel de ingresos, educación y nivel de vida y, por lo tanto, estos resultados demuestran que es el grupo socioeconómico con mayor capacidad para decidir dónde vivir en la ciudad, facilidad que explota para separarse espacialmente del resto de la sociedad, al menos en términos residenciales. Después de este cluster, el siguiente cluster que presenta niveles más altos de segregación es quizá el Cluster 2, aquí denominado Oficinistas en Bloques, que como su nombre indica ocupa un menor espacio en la ciudad y de ahí su nivel más alto en el índice de Concentración Absoluta (ACO) (0.85), y su homogeneidad interna representada por un muy alto índice de disimilaridad (0.82). El Cluster 3, denominado Proletariado Periférico, presenta niveles medio-altos de segregación en todos los índices, y el nivel más alto en la dimensión de clustering, es decir, es el que se encuentra más agrupado espacialmente en zonas contiguas donde el mismo cluster está presente a lo largo de la periferia. El Cluster 5, denominado Zonas Mezcladas, presenta valores altos para D y ACO, aunque muy bajo nivel de aislamiento $(\mathrm{Px})$, sin embargo, tal y como se mencionó anteriormente, estos resultados disfrazan la heterogeneidad interna de las amplias manzanas que componen este cluster, cuyos habitantes podrían pertenecer perfectamente a uno u otro cluster, por lo que sus índices han de ser tomados con precaución.

CUADRO 3 | Tamaños de los seis clusters seleccionados

\begin{tabular}{|c|c|c|c|c|c|}
\hline CLUSTER & $\begin{array}{c}\text { D } \\
\text { DISIMILARIDAD }\end{array}$ & $\begin{array}{c}\text { PX } \\
\text { AISLAMIENTO }\end{array}$ & $\begin{array}{c}\text { ACO } \\
\text { CONCENTRACION } \\
\text { ABSOLUTA }\end{array}$ & $\begin{array}{c}\text { ACL } \\
\text { CLUSTERING }\end{array}$ & H ENTROPÍA \\
\hline Cluster 1: Periferia rural marginal & 0,75 & 0,54 & 0,6 & 0,34 & 0,51 \\
\hline Cluster 2: Oficinistas en bloques & 0,82 & 0,63 & 0,85 & 0,34 & 0,63 \\
\hline Cluster 3: Proletariado periférico & 0,65 & 0,69 & 0,52 & 0,52 & 0,41 \\
\hline Cluster 4: Elites envejecidas & 0,86 & 0,71 & 0,77 & 0,46 & 0,68 \\
\hline Cluster 5: Zonas mezcladas & 0,83 & 0,38 & 0,71 & 0,17 & 0,52 \\
\hline Cluster 6: Clase media educada & 0,66 & 0,59 & 0,8 & 0,4 & 0,42 \\
\hline
\end{tabular}

FUENTE ELABORACIÓN PROPIA. 
Finalmente, en el Cuadro 4 se presenta una matriz con los índices de disimilitud calculados para cada par de clusters, reflejando cuán segregado está un cluster de otro (anteriormente se habían calculado entre cada cluster y el resto de la población). Como puede observarse, las parejas de clusters más segregadas entre sí son los clusters 4 y 1 , seguidos por 4 y 3,1 y 2 , y 1 y 6 . Es decir, mientras es de esperar que el Cluster 1, Periferia Rural Marginal, esté separado de los demás por su ubicación en el borde de la ciudad, sorprende de nuevo el alto grado de segregación del Cluster 4, Élites Urbanas, con respecto a todos los demás cluster, excepto quizá con el Cluster 6, Clase Media Educada, el único con el que el Cluster 4 presenta un índice $D$ ligeramente por debajo de 0.80 . En el extremo opuesto de las relaciones de segregación, las parejas de clusters que menor segregación presentan entre sí son el 1 y 3 , seguidos por el 3 y 6, y 4 y 6 . Es decir, los clusters menos segregados con otros son el 3, Proletariado Periférico y 6 Clase Media Educada, los cuales además, en los mapas de las Figuras 5, 6 y 7 ocupan una distribución geográfica más repartida por toda la ZMCM.

CUADRO 4 | Matriz de índices de disimilitud entre pares de clusters

\begin{tabular}{lcccccc}
\hline Cluster & Cluster & Cluster & Cluster & Cluster & Cluster & Chuster \\
& $\mathbf{1}$ & $\mathbf{2}$ & $\mathbf{3}$ & $\mathbf{4}$ & $\mathbf{5}$ & $\mathbf{6}$ \\
\hline Cluster 1: Periferia rural marginal & & & & & \\
\hline Cluster 2: Oficinistas en bloques & $\mathbf{0 , 9 3}$ & & & \\
\hline Cluster 3: Proletariado periférico & 0,65 & 0,87 & & \\
\hline Cluster 4: Elites envejecidas & $\mathbf{0 , 9 8}$ & 0,88 & $\mathbf{0 , 9 5}$ & \\
\hline Cluster 5: Zonas mezcladas & $\mathbf{0 , 9}$ & 0,89 & 0,84 & 0,87 & \\
\hline Cluster 6: Clase media educada & $\mathbf{0 , 9 1}$ & 0,82 & 0,72 & 0,79 & 0,81 \\
\hline
\end{tabular}

En negrita se señalan los índices más altos $(>=0.90)$ y en itálica los más bajos $(<=0.80)$.

FUENTE ELABORACIÓN PROPIA.

\section{Conclusión}

Los escasos trabajos que han medido el nivel de segregación residencial para áreas pequeñas en México han reducido la variabilidad socioeconómica de la población a dos o tres dimensiones mediante análisis factorial o de componentes principales. En estos estudios los índices de segregación se miden de manera separada para cada uno de estos componentes o, en casos, para cada variable censal analizada. El problema con esta estrategia es la pérdida de información clave en cuanto a las relaciones entre dichas variables, que tomadas por sí solas no reflejan necesariamente la compleja configuración de estilos de vida y perfil sociodemográfico de las vecindades que componen la ciudad. Es por esto que en este estudio hemos propuesto la utilización de una nueva metodología para la clasificación de zonas de la ciudad en 
grupos homogéneos de estilos de vida sociodemográficos mediante las técnicas de clustering para crear una clasificación geodemográfica. Para ello hemos tomado la manzana como la unidad básica de análisis que mejor representa las relaciones sociales del entorno inmediato de los individuos y hogares y los patrones creados por los complejos procesos del mercado de vivienda. Como resultado hemos propuesto una novedosa tipología de seis clusters que podríamos resumir como "geo-sociodemográficos", y que no corresponden necesariamente a ninguna escala ordinal en cuanto a su dimensiones aisladas de ingresos, educación, ocupación y vivienda, sino que simplemente reflejan distintas configuraciones de lo que, a falta de un mejor término y siguiendo la literatura del geomárketing, podríamos denominar "estilos de vida". Los factores que parecen influir en la configuración de los clusters obtenidos automáticamente reflejan los elementos clásicos en geografía urbana para distinguir tipologías residenciales: nivel de educación, ingresos, ocupación, estructura de edad, configuración de hogar-vivienda y grado de centralidad en la ciudad, en este caso medidos de manera combinada.

Es, por tanto, esta tipología de manzanas clasificadas en estilos de vida la que ha servido como unidad base para el cálculo de los índices de segregación descritos en la última sección. Una vez más, recalcamos que los resultados obtenidos, si bien son concluyentes a la vez que esclarecedores, se refieren al nivel de segregación residencial entre "tipos de manzanas" o clusters geodemográficos, no de individuos ni hogares.

La evidencia aquí encontrada es que el grupo más segregado en la ZMCM es el más acomodado socioeconómicamente (Élites Urbanas). Además, tras calcular cinco diversos índices de segregación para medir algunas de sus múltiples dimensiones, esta afirmación se sigue sosteniendo claramente. En términos de la distribución espacial de los grupos, se apreció que algunos de ellos presentan rasgos de distribución más acordes con el modelo tradicional de segregación residencial, tal es el caso del cluster Élites Urbanas, que se presenta muy concentrado hacia el poniente de la ciudad tanto en el Distrito Federal en localizaciones tradicionales, como en el Estado de México en nuevas zonas residenciales. También las clases medias permanecen muy concentradas en la parte central de la zona urbana y el suburbio interior. Por otra parte, y más acorde con el modelo reciente de la dispersión urbana y la fragmentación del espacio, es notoria la difusión de los clusters Proletariado Periférico y Periferia Urbano-Rural Marginal, este último con una localización más periférica que el primero; el cluster Empleados de Oficina en Unidades Habitacionales tiende a localizarse en los intersticios urbanos, pero sobre todo a lo largo de los principales accesos carreteros de la ciudad, como, por ejemplo, en las carreteras hacia las ciudades de Puebla, Pachuca y Querétaro. En conjunto, es notable esta combinación de zonas más homogéneas (élites y clases medias), y las áreas con patrones de fragmentación que se destacan sobre todo en una franja al norte del Distrito Federal y otra franja al sur del Distrito Federal con una mayor mezcla de estratos sociodemográficos. Es decir, en la ZMCM se han encontrado trazas tanto del nuevo modelo de segregación residencial en América Latina como del modelo tradicional (Janoschka, 2002). Esto puede estar indicando la presencia de 
un proceso de transición urbana que es necesario estudiar con mayor profundidad y de manera diacrónica en el tiempo para poder establecer tendencias en la segregación. Por diversas limitaciones, el presente trabajo se ha reducido a estudiar un solo Censo de población, por lo que no hemos podido establecer dichas tendencias diacrónicas empíricamente. No obstante, esperamos que la aportación de una nueva metodología de análisis geodemográfico presentada en este trabajo sea desarrollada en futuros estudios urbanos en América Latina. IEURE

\section{Referencias bibliográficas}

Alegría, T. (1994). Segregación socioespacial urbana. El ejemplo de Tijuana. Estudios Demográficos y Urbanos, Vol. 9, No. 2, El Colegio de México, pp. 411-428.

Apparicio, P.; Petkevitch, V. \& Charron, M. (2008). Segregation Analyzer: a C\#.Net application for calculating residential segregation indices. Cybergeo, No. 414.

Ariza, M. \& Solís, P. (2009). Dinámica Socioeconómica y Segregación Espacial en Tres Areas Metropolitanas de México, 1990 y 2000. Estudios Sociológicos XXVII, 79, pp. 171-209.

Borsdorf, A. (2003). Cómo Modelar el Desarrollo y la Dinámica de la Ciudad Latinoamericana. Revista EURE, Vol. 29, No. 86.

Cabrales, L. F. (2002). Nuevas Formas y Viejos Valores: urbanizaciones Cerradas de Lujo en Guadalajara. En L. F. Cabrales (ED.) Latinoamérica: Paises Abiertos, Ciudades Cerradas. México: Universidad de Guadalajara, UNESCO.

Caldeira, T. P.R. (2000). City of Walls. Crime, Segregation and Citizenship in Sao Paulo. Los Angeles, London: University of California Press.

Cáceres, G. \& Sabatini, F. (2004). (Eds.). Barrios Cerrados en Santiago de Chile. Entre la Exclusión y la Integración Residencial. Santiago: Lincoln Institute of Land Policy, Pontificia Universidad Católica de Chile.

Castells, M. (1977). The Urban Question. A Marxist Approach. London: Edward Arnold.

Ciccolella, P. (1999). Globalización y Dualización en la Región Metropolitana de Buenos Aires. Grandes Inversiones y Reestructuración Socioterritorial en los años noventa. Revista EURE, Vol. XXV, No. 76, pp.5-27.

Ciccolella, P. \& Baer, L. (2008). Buenos Aires tras la Crisis: ¿Hacia una Metrópolis más integradora o más excluyente? Ciudady Territorio-Estudios Territoriales, Vol XL, No. 158, pp. 641-660.

Cortés, S. (2008). "Vergüenza de Vivir donde Vivo”: Ideas para una Re-Conceptualización de la Segregación Residencial Socioeconómica. Revista de Antropología Americana, Vol. 3, No. 3, pp. 419-445.

CONAPO (1998). Escenarios demográficos y urbanos de la zona metropolitana de la ciudad de México, 1990-2010. México D.F: Consejo Nacional de Población.

De Mattos, C. (1999). Santiago de Chile, Globalización y Expansión Metropolitana: lo que existía sigue existiendo. Revista EURE, Vol. XXV, No. 76, pp. 29-56.

Dickinson, F.; García, A. \& Pérez, S. (1999). Social Differentiation and Urban Segregation in a Mexican Regional Metropolis. En A. G. Aguilar \& I. Escamilla (Eds.). Problems of Megacities: Social Inequalities, Environmental Risk and Urban Governance. México: Instituto de Geografía, UNAM. 
Duhau, E. (2003). División Social del Espacio Metropolitano y Movilidad Residencial. Papeles de Población, No. 36, pp. 161-210.

Duncan, O. \& Duncan, B. (1955). A Methodological Analysis of Segregation Indices. American Sociological Review, No. 20, 210-217.

Ford, L. (1996). A New and Improved Model of Latin American City Structure. The Geographical Review, Vol. 86, No. 3, pp. 437-440.

Griffin, E. \& Ford, L. (1980). A Model of Latin American City Structure. Geographical Review, Vol. 70, No. 4, pp. 397-422.

González, S. \& Villeneuve, P. (2007). Transformaciones en el Espacio Sociorresidencial de Monterrey, 1990-2000. Estudios Demográficos y Urbanos, Vol. 22, No. 1, El Colegio de México, pp. 143-178.

Harris, R.; Sleight, P. \& Webber, R. (2005). Geodemographics: neighbourhood targeting and GIS. Chichester, UK: John Wiley and Sons.

Hernández, E. (2001). Globalización y Segregación Urbana en Tijuana, Baja California. Comercio Exterior, marzo, pp. 234-242.

Ickx, W. (2002). Los Fraccionamientos Cerrados en la Zona Metropolitana de Guadalajara. En L. F. Cabrales (Ed.) Latinoamérica: Paises Abiertos, Ciudades Cerradas. México: Universidad de Guadalajara, UNESCO.

Instituto Nacional de Estadística y Geografía (INEGI) (2000). Distrito Federal, XII Censo General de Población y Vivienda. Aguascalientes: Instituto Nacional de Estadística y Geografía.

Instituto Nacional de Estadística y Geografía (INEGI) (2000). Estado de México, XII Censo General de Población y Vivienda. Aguascalientes: Instituto Nacional de Estadística y Geografía.

Johnston, R. J. (1980). City and Society. An outline for Urban Geography. Great Britain: Penguin Books. Janoschka, M. (2002). El Nuevo Modelo de la Ciudad Latinoamericana: Fragmentación y Privatización. Revista EURE, Vol XXVIII, No. 85, pp. 11-29.

Kaplan, D. H.; Wheeler, J. O. \& Holloway, S. R. (2004). Urban Geography. USA: John Wiley and Sons. Knox, P. \& Pinch, S. (2000). Urban Social Geography. An Introduction. England: Prentice Hall.

Latham et al (2009). Key Concepts in Urban Geography. London: SAGE publications.

Lefebvre, H. (Kofman \& Lebas Eds.) (1996). Writings on cities. Oxford: Blackwell.

Longley, P. A. \& Mateos, P. (2006). Un nuevo y prominente papel de los SIG y Geomárketing en la provisión de servicios públicos. Geofocus, No. 5, pp.1-5.

Marcuse, P. (1997). The Enclave, The Citadel, and The Ghetto. What has changed in the Post-Fordist U.S. City. Urban Affairs Review, Vol. 33, No. 2, pp. 228-264.

Marcuse, P. (1993). What's so new about divided cities? International Journal of Urban and Regional Research, Vol. 17, No. 3, pp. 355-365.

Massey, P. \& Denton, K. (1988). The Dimensions of Residential Segregation. Social Forces, No. 67, pp. 281-315.

Park, R. E.; Burgess, E. W. \& McKenzie, R. D. (1970). The City. USA: The University of Chicago Press.

Massey, D.; White, M. \& Phua, V. (1996). The Dimensions of Segregation Revisited. Sociological Methods and Research, No. 24.

Oehmichen, C. (2001). Espacio Urbano y Segregación Etnica en la Ciudad de México. Papeles de Población, No. 28, CIEAP/UAEM, pp. 181-197. 
Ortiz, J. \& Schiappacasse, P. (2000). Evolución de la Diferenciación Areal Interna del Espacio Social del Gran Santiago: una dinámica opuesta a la sostenibilidad social de la ciudad. Investigaciones Geográficas, No. 34, pp. 61-76.

Pacione, M. (2009). Urban Geography. A Global Perspective. London, New York: Routledge.

Robinson, G. M. (1998). Methods and Techniques in Human Geography. Chichester: John Wiley and Sons.

Rodríguez, I. \& Mollá, M. (2002). Urbanizaciones Cerradas en Puebla y Toluca. En L. F. Cabrales (Ed.). Latinoamérica: Paises Abiertos, Ciudades Cerradas. México: Universidad de Guadalajara, UNESCO.

Rodríguez, J. (2001). Segregación Residencial Socioeconómica: ¿Qué es?, ¿Cómo se mide?, ¿Qué está pasando?, ¿Importa? Serie Población y Desarrollo, No. 16, CEPAL, Naciones Unidas, Santiago de Chile.

Rodríguez, J. \& Arriagada, C. (2004). Segregación Residencial en la Ciudad Latinoamericana. Revista EURE, Vol. 30, No. 89, pp. 5-24.

Rubalcava, R. M. \& Schteingart, M. (2000a). Segregación Socioespacial. En G. Garza (Ed.). La Ciudad de México en el fin del Segundo Milenio. México: Gobierno del Distrito Federal, El Colegio de México.

Rubalcava, R. M. \& Schteingart, M. (2000b). La División Social del Espacio en las Grandes Metrópolis Mexicanas. Un estudio comparativo. El Mercado de Valores, abril, pp. 20-33.

Sabatini, F.; Cáceres, G. \& Cerda, J. (2001). Segregación Residencial en las Principales Ciudades Chilenas: Tendencias en las Tres Últimas Décadas y Posibles Cursos de Acción. Revista EURE, Vol. XXVII, No. 82, pp. 21-42.

Sabatini, F. (2003). La Segregación Social del Espacio en las Ciudades de América Latina. Washington D.C.: Banco Interamericano de Desarrollo.

Sabatini, F. \& Cáceres, G. (2004). Los Barrios Cerrados y la Ruptura del Patrón tradicional de Segregación en las Ciudades Latinoamericanas: el caso de Santiago de Chile. En G. Cáceres y F. Sabatini (Eds.). Barrios Cerrados en Santiago de Chile. Entre la Exclusión y la Integración Residencial. Santiago: Lincoln Institute of Land Policy, Pontificia Universidad Católica de Chile.

Sabatini, F. \& Brain, I. (2008). en Social Urbana: Mitos y Claves. Revista EURE, Vol. XXXIV, No. 103, pp. 5-26.

Safa, P. (2002). Construir Mundos, Levantar Muros y Preservar Patrimonios: Condominios y Fraccionamientos Cerrados en la Ciudad de México. En L. F. Cabrales (Ed.). Latinoamérica: Países Abiertos, Ciudades Cerradas. México: Universidad de Guadalajara, UNESCO.

Saraví, G. A. (2008). Mundos Aislados: Segregación Urbana y Desigualdad en la Ciudad de México. Revista EURE, Vol. XXXIV, Núm. 103, pp. 93-110.

SEDESOL, CONAPO, INEGI (2007). Delimitación de las Zonas Metropolitanas de México 2005. México: Secretaría de Desarrollo Social, Consejo Nacional de Población, Instituto Nacional de Estadística, Geografía e Informática.

Schteingart, M. (2001). La División Social del Espacio en las Ciudades. Perfiles Lationoamericanos, No. 19, pp. 13-31.

Shevky, E \& Bell, W. (1955). Social Area Analysis. Stanford CA: Stanford University Press.

Simpson, E. H. (1949). Measurement of diversity. Nature, Vol. 163, No. 688.

Solinis, G. (2002). Introducción. En L.F. Cabrales (Ed.). Latinoamérica: Paises Abiertos, Ciudades Cerradas. México: Universidad de Guadalajara, UNESCO. 
Vargas, M. \& Royuela, V. (2006). Segregación Residencial. Una Revisión de la Literatura. Faculty of Economics and Business, Universidad Diego Portales, Chile.www.udp.cl/economiayempresa/ academicos/docs/RLSegregacionResidencial_DT_14.pdf (accessed 17 $7^{\text {th }}$ September 2009).

Vickers, D. \& Rees, P. (2007). Creating the UK National Statistics 2001 output area classification. Journal of the Royal Statistical Society: Series A (Statistics in Society) 170(2): pp. 379-403.

Young, I. M. (2002). Residential Segregation and Regional Democracy. Inclusion and Democracy, Oxford University Press, Oxford, 196-235. 